\title{
SWIR Images Evaluation for Pedestrian Detection in Clear Visibility Conditions
}

\author{
Alina Miron, Abdelaziz Bensrhair \\ INSA de Rouen \\ 685 Avenue de l'Université \\ 76801 Saint-Étienne-du-Rouvray, FRANCE \\ \{alina.miron, \\ abdelaziz.bensrhair\} einsa-rouen.fr
}

\author{
Rean I. Fedriga, Alberto Broggi \\ Dipartimento di Ingegneria dell'Informazione \\ Università di Parma \\ Parco Area delle Scienze 181/A \\ 43124 Parma, ITALY \\ ffedriga, broggi\}@vislab.it
}

\begin{abstract}
Pedestrian detection is a difficult task with significant practical interests especially in the field of Intelligent Transportation Systems. While the problem has been thoroughly studied from a visible, mid-wave infrared and far-wave infrared cameras perspective, little attention has been paid to the short-wave infrared spectrum. But although light in the short-wave infrared region is imperceptible to the eye, due to its reflective nature, it interacts with objects similarly to the visible wavelengths. As a result, short-wave images, including both shadows and contrast, are comparable to visible images in resolution and detail but they are not comparable in color, a feature which makes objects recognizable and yields one of the advantages of the short-waves, namely object or individual identification. In this paper we evaluate the pertinence of employing the short-wave bandwidths for the task of detecting pedestrians in clear visibility conditions.
\end{abstract}

\section{INTRODUCTION}

If self-driven vehicles were the norm, car travel would assume a new identity as a type of private transportation that incorporates aspects of public transportation with, as its biggest benefit, the most important thus difficult to achieve: people's safety.

Despite the number of scientific works dealing with pedestrian detection for Advanced Driver Assistance Systems (ADAS), to this day for many reasons [1], [2] the problem has yet to be solved. People appear in highly cluttered backgrounds and have a wide range of appearances, due to body size and pose, clothing and outdoor lighting conditions. Because the vehicle is moving there is no possibility to use simple background subtraction methods (such as those for surveillance applications) to obtain a foreground region containing the human shape. Furthermore, pedestrians can exhibit a highly irregular motion, making prediction and situation analysis difficult. Finally, there are hard real-time requirements and tight performance criteria.

The problem of pedestrian detection has been approached from both a hardware and software perspective, using different sensors and developing many different detection techniques. From a hardware perspective there are active and passive sensors. For the active sensors category those that currently give the best results are the laser sensors, especially LIDARs ([3], [4]), which still remain too costly for ADAS systems. The passive sensors category includes all cameras based systems. The most frequently used are visible cameras either in a monocular ([1], [5], [6]) or a stereo configuration ([7], [8], [2]). Infrared cameras (mainly working in the thermal spectrum) have too been successfully employed for pedestrian detection systems ([9], [10], [11], [12], [13]). An extended review of different pedestrian detection systems for ADAS based on a variety of sensors is given in [14]. A variety that however does not include short-wave sensors, able to provide images with a noticeably different information content from visible ones (see fig. 1).

Imaging in the Short Wave InfraRed (SWIR) can bring useful contrast to situations and applications where visible or thermal imaging cameras are ineffective. This makes SWIR frequently used for diverse applications such as aligning telecommunications fibers and sources, engineering optical wave-guides, inspecting pharmaceutical quality, sorting recycled plastics, monitoring incoming sources of raw agricultural products to groom out contamination by dirt, stones or packaging debris, as well as grade sorting by moisture level or fat content [15]. Unlike Mid Wave IR (MWIR) and Long Wave IR (LWIR), SWIR cameras can image through the windshield and thus be mounted in the vehicle's cabin for a "driver's eye" view of the way ahead. Moreover, SWIR imagers have the ability to see clearer at long distance through the atmosphere, making SWIR suitable for investigations in the automotive field [16] the main issues having been to achieve low cost SWIR sensors operating at close to room temperature and CMOS compatible. In the last few years though the standard technology based on indium bump hybridization has progressively decreased its pixel pitch going to VGA or XVGA format mainly available in the US. Nevertheless, most of VGA format were based on the $25 \mu \mathrm{m}$ pitch from FLIR Indigo ISC0002 readout circuit which consists in an expensive device not compatible with the automotive market.

Research done within the EU funded 2WIDE_SENSE Project [17], project of which this work is part, has involved a $15 \mu \mathrm{m}$ pitch format - the best one to replace the $\frac{1}{4}$ VGA format - reaching a low cost hybridization process and packaging to obtain a SWIR imager most suitable for the automotive market. Within the 2WIDE_SENSE Project the characteristics of SWIR images for pedestrian detection have been explored (see fig. 1): in difficult visibility conditions as haze and fog [18] and in clear visibility conditions for 
the scope of this work. Purpose of this article is to illustrate our tests carried out in clear visibility conditions comparing different classification algorithms for the pedestrian detection task, after having evaluated if features learned on visible images are suitable to be used on SWIR images. Approaches tested include an SVM classifier based on deformable part models ([19], [20]), on grammar models [21] and a HAAR based classifier [22].

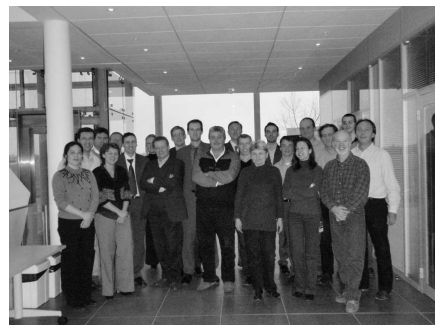

visible

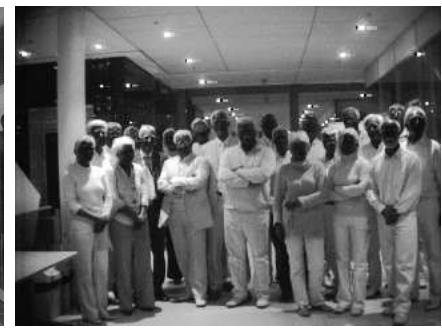

SWIR
Fig. 1. Images acquired indoor: visible and SWIR bandwidths highlight different features both for people and background.

Section II of this paper briefly introduces the equipment employed to acquire the images, then in section III the characteristics of SWIR images are discussed and in section IV the dataset created for the tests is described. Section V reports the results obtained from our tests with different state-ofthe-art classifying algorithms and section VI states our final considerations and proposals for further investigations.

\section{HARDWARE EQUIPMENT}

The device employed to acquire the visible and SWIR images shown in this work was totally developed within the 2WIDE_SENSE project. Producing a low-cost camera able to acquire in the full visible to SWIR bandwidth required the development of a specific sensor and of a large bandwidth lens (see fig. 2). In addition, the camera features a Bayer-like four filter pattern on its Focal Plane Array (FPA) to enable the simultaneous and independent acquisition of four images, each one in a different spectral bandwidth (see fig. 3 and 4).
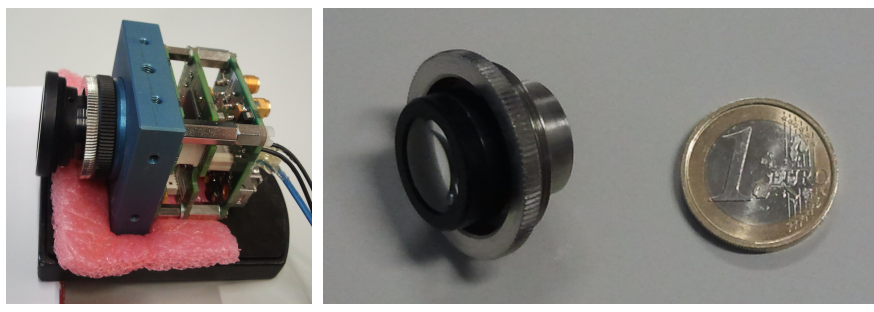

Fig. 2. The full-VGA sensor camera module and micro-lens.

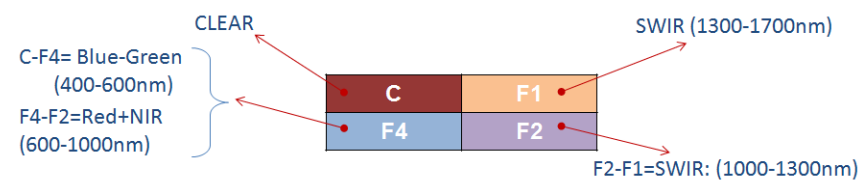

Fig. 3. The $4 \times 4$ filter mask applied on the FPA.

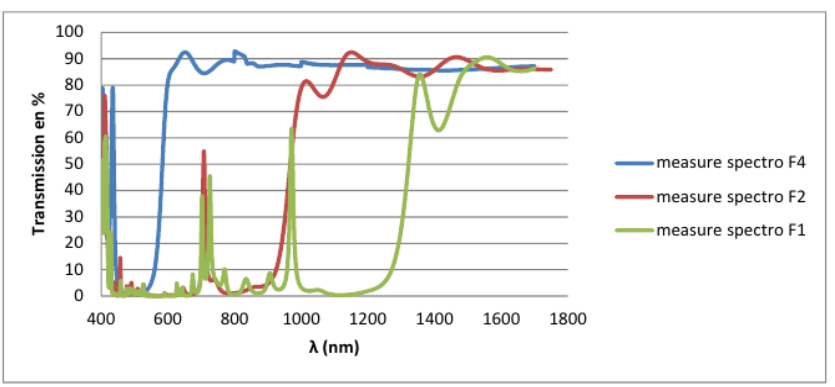

Fig. 4. Filters F1, F2 and F4 transmission bands.

The four high-pass filter bandwidths $\mathrm{C}$ (stands for Clear, acquires the full lens spectrum), F1, F2, F4 have been selected according to most used ADAS functions. For example filter F4 isolates the red bandwidth useful to find prohibitory/warning signs for Traffic Sign Recognition and preceding vehicles back lights for High Beam Assist. However, other bandwidths not included in the pattern may be obtained combining the 4 filters contributions, e.g. the blue and green bandwidth as a difference between $\mathrm{C}$ and $\mathrm{F} 4$.

\begin{tabular}{ll}
\hline Characteristic & Value \\
\hline Spectral Range & VIS / NIR / SWIR \\
Filter Pattern & C $400 \div 1700 \mathrm{~nm}$ \\
& F4 $540 \div 1700 \mathrm{~nm}$ \\
& F1 $1300 \div 1700 \mathrm{~nm}$ \\
Dynamic Range & $120 \mathrm{~dB}$ \\
Angular Resolution & min $11 \mathrm{px} /{ }^{\circ}$ \\
Field of View & HFOV $30^{\circ}$ \\
& VFOV $22^{\circ}$ \\
Imager Resolution & $640 \times 512 \mathrm{px}$ \\
Pixel Pitch & $15 \mu \mathrm{m}$ \\
Focal Length & $18 \mathrm{~mm}$ \\
Frame Rate & $>24 \mathrm{fps}$ \\
Camera Size & $130 \times 40 \times 40 \mathrm{~mm}$ \\
Camera Weight & $500 \mathrm{gr}$ \\
Temperature Range & $-40 \div 80^{\circ} \mathrm{C}$ \\
Supply Voltage & $6 \div 16 \mathrm{~V}$ \\
Power Consumption & $<1 \mathrm{~V}$ \\
\hline \multicolumn{2}{c}{ CABLE I } \\
\end{tabular}

The camera module sensor, mainly developed by AlcatelThales III-V Lab, is an uncooled InGaAs and InP-based $640 \times 512 p x$ array with a $15 \mu \mathrm{m}$ pitch and a MAGIC ${ }^{\mathrm{TM}}$ logarithmic readout circuit. The two main features of the sensor are the large spectrum sensitivity $(400-1700 \mathrm{~nm})$ and the logarithmic gain that enables to avoid saturation effects. Table I shows a schematic overview of the camera module characteristics.

The OB-V-SWIR_16 apochromatic micro-lens module has been developed by project partner OPTEC to let the camera 
exploit the full spectrum capabilities and features an almost constant transmittance in its whole functioning bandwidth: $>98 \%$ in the $400-1550 \mathrm{~nm}$ interval and decreasing down to $96 \%$ in the $1550-1700 \mathrm{~nm}$.

Further details about sensor and lens are reported in [18] and at the 2WIDE_SENSE Project website [17].

Tests described in this paper involve filters C, F1 and F2 excluding filter F4 the operating bandwidth of which was outside the scope of this work. Moreover in this phase of the project the camera module final prototype with the four filters on the FPA was not yet available so the filters had to be manually switched and applied to the lens.

\section{SWIR IMAGE ANALYSIS}

From an empirical perspective, visible and SWIR images acquired indoor highlight some very different characteristics (e.g. fig. 1 and 5), but as soon as acquisitions are moved outdoor the span of those differences reduces significantly (fig. 6).

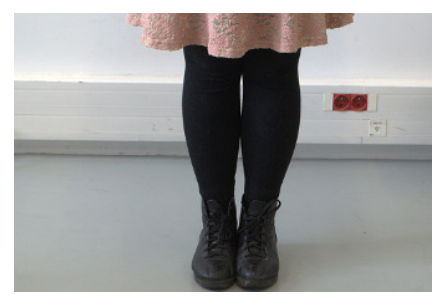

(a)

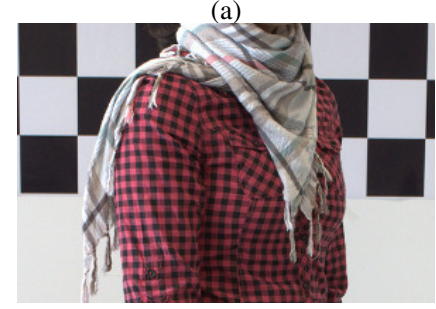

(c)

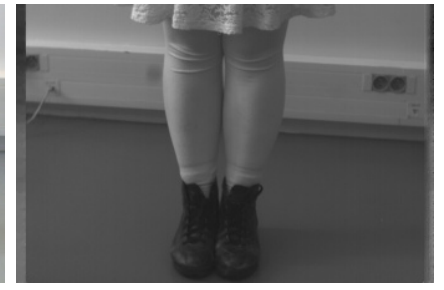

(b)

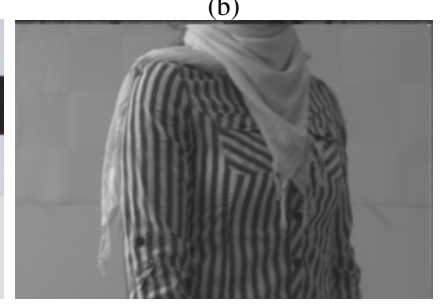

(d)
Fig. 5. Indoor image examples of how clothing appears differently between visible [a, c] and SWIR spectra [b, d]. Appearance in the SWIR is influenced by the materials composition and dyeing process.

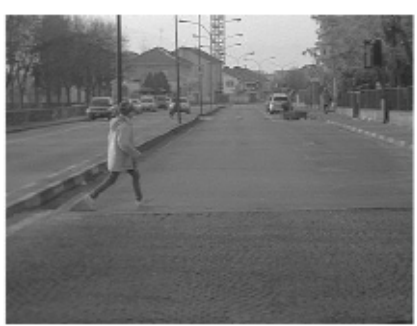

visible

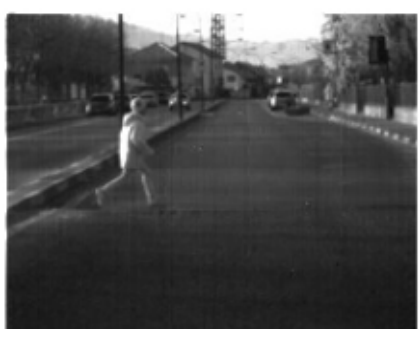

SWIR
Fig. 6. Images acquired outdoor: visible and SWIR bandwidths highlight similar features both for pedestrian and background.

There may still be some distinction in the appearance of clothes and human skin, but people have a similar aspect from their edges gradients point of view. Fig. 7 shows a visualisation of Haar wavelengths computed on the diagonal, horizontal and vertical directions along with results after Sobel filtering (the basis for our gradient computation of HOG features as will be explained in section V) for the same scene acquired through filters C, F1 and F2. From the computed features only small differences can be observed in the hair, clothes and background, the main contours of the objects remaining quite similar for both Haar and Sobel transformations in the different bandwidths tested.

\section{Dataset OVERView}

Corresponding to filters $\mathrm{C}, \mathrm{F} 1$ and $\mathrm{F} 2$, three image sequences were acquired choosing a fixed setup for the camera in order to compare the results obtained using different bandwidth filters for similar scenes. In this phase of the project the filters had to be manually changed for each acquisition therefore some small differences in the scenes can be expected. The number of full-frame images tested for each bandwidth are presented in table II.

Following the sequences acquisition, a total of 4348 Bounding Boxes (BB) surrounding pedestrians have been manually annotated, out of which only $4.57 \%$ are occluded. This corresponds to 1998,1200 and $1150 \mathrm{BB}$ annotated in filter $\mathrm{C}$, filter F2 and filter F1 bandwidths respectively.

In fig. 8 the annotated pedestrians height distribution in each sequence is shown. It can be observed that most of the BB stand in a medium [50-100) or close range [100200] distance with respectively $41 \%$ and $48 \%$ of the total BB. The nearest pedestrians (with $200 p x$ as average height) are placed $4 \mathrm{~m}$ away from the acquisition system while the farthest (about $50 \mathrm{px}$ tall) are $30 \mathrm{~m}$ far.

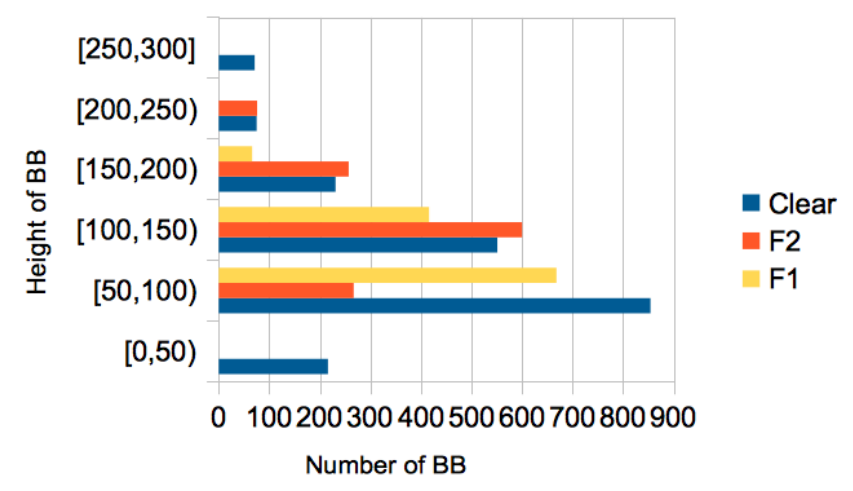

Fig. 8. Height distribution over the annotated pedestrians.

\begin{tabular}{llll}
\hline Bandwidth & Clear & F2 & F1 \\
\hline Full-frame Images & 1704 & 1374 & 1421 \\
\hline \multicolumn{5}{c}{ TABLE II } \\
\end{tabular}

NUMBER OF FULL-FRAME IMAGES ON EACH TESTED BANDWIDTH 


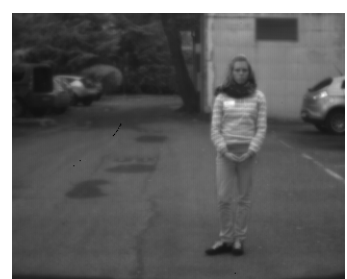

(a1)

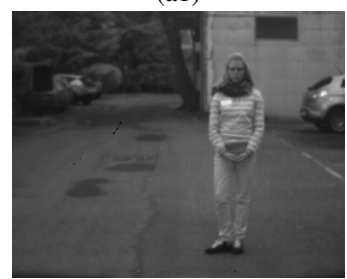

(a2)

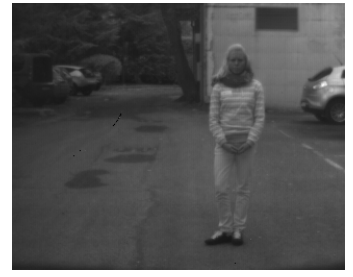

(a3)

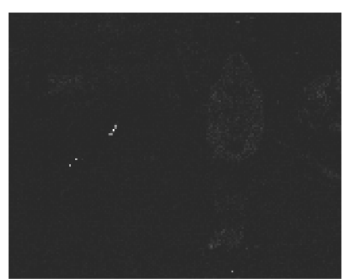

(b1)

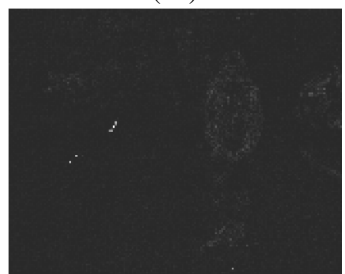

(b2)

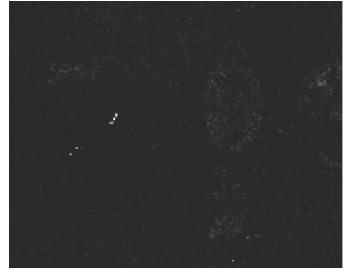

(b3)

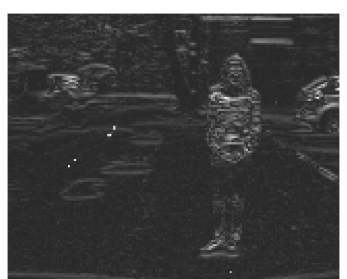

(c1)

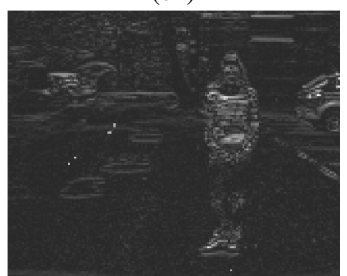

(c2)

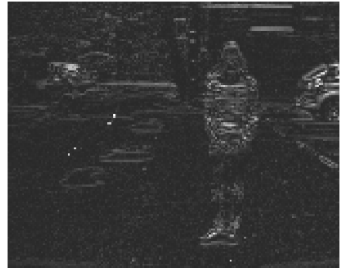

(c3)

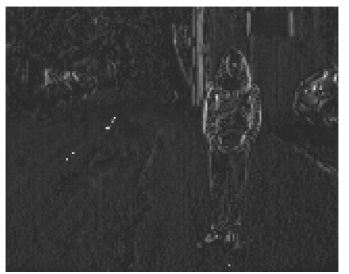

(d1)

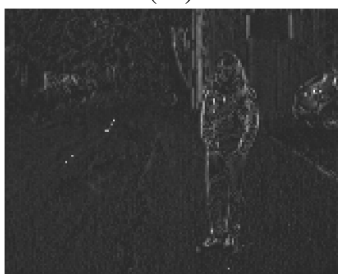

(d2)

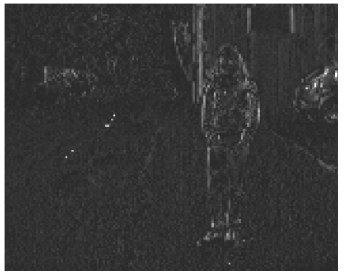

(d3)

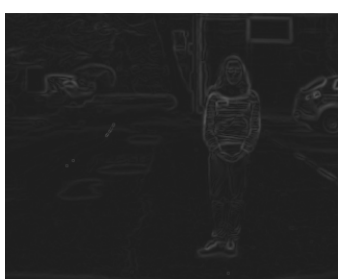

(e1)

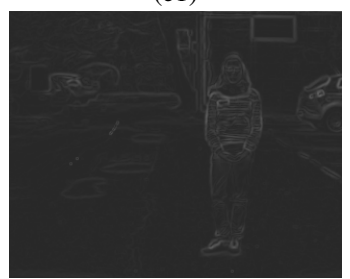

(e2)

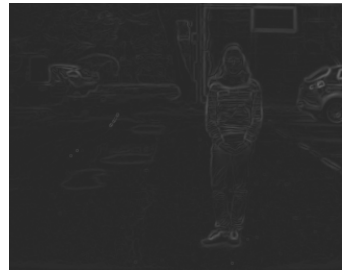

(e3)

Fig. 7. Image comparison between visible range [a1], F2 filter range [a2] and F1 filter range [a3] with the corresponding on-column visualization of HAAR wavelets: diagonal [b1, b2, b3], horizontal [c1, c2, c3], vertical [d1, d2, d3] and Sobel filter [e1, e2, e3]. Due to negligeble values of the HAAR wavelet features along the diagonal direction, the corresponding images [b1, b2, b3] appear very dark.

TABLE III

RESULTS OF HOG CLASSIFIER ON BB

\begin{tabular}{|l|l|l|l|}
\cline { 2 - 4 } \multicolumn{1}{c|}{} & C & F2 & F1 \\
\hline Precision(\%) & 95.18 & 94.79 & 93.85 \\
Recall(\%) & 59.00 & 88.12 & 76.92 \\
F-measure(\%) & 72.84 & $\mathbf{9 1 . 3 3}$ & 84.54 \\
\hline
\end{tabular}

\section{EXPERIMENTS}

\section{A. Features from Visible to SWIR}

Most of the top algorithms developed for images acquired in the visible bandwidth employ Histogram of Oriented Gradients (HOG) features [23], usually by combining them with others as HAAR-like features [24], color self-similarity [25], etc. In order to see if features trained on visible images are suitable to use for the SWIR images, we have trained an SVM classifier based on HOG features using the INRIA image dataset $^{1}$ as source.

The classifier was tested on all the acquired sequences over the annotated $\mathrm{BB}$ as positive examples and randomly selected negative $\mathrm{BB}$ from the images. The number of negative $\mathrm{BB}$ is taken to be twice the number of positives. As table III shows, the detection precision is good for all the filters tested while a bigger difference results in the recall values.

\section{B. Pedestrian Detection Evaluation}

Having verified that features learned from the visible spectrum can be applied to the SWIR, the pedestrian detectors

\footnotetext{
${ }^{1}$ http://pascal.inrialpes.fr/data/human/
}

evaluation has been undertaken. Three have been the pedestrian detectors chosen for testing: deformable part models [19], [20], grammar models [21] and HAAR based classifier [22]. Being most of the annotated pedestrians available in our sequences at a medium or close range distance, the classifiers based on deformable part models and grammar models were theroetically the most suitable for the task [21]. The third classifier was included to evaluate the performance of other state-of-the-art features as the HAAR-like ones. The classifiers were all trained on the INRIA dataset.

A detected $\mathrm{BB}\left(B B_{d t}\right)$ is considered to be a true positive if it overlaps with a ground truth BB $\left(B B_{g t}\right)$ for at least $50 \%$ of its surface (Pascal measure as used in [1], see eq. 1).

$$
\frac{\operatorname{area}\left(B B_{d t} \cap B B_{g t}\right)}{\operatorname{area}\left(B B_{d t} \cup B B_{g t}\right)}>0.5
$$

Results obtained for the three different filters are presented in table IV, varying depending on the classification rules and the particular scene.

The part based classifier gives better results on scenes acquired with filters $\mathrm{C}$ and $\mathrm{F} 1$, while with the grammar based classifier better results are obtained on F2 filtered images. Examples of pedestrian detection results with all the algorithms tested are presented in fig. 10.

An additional test considering only pedestrians more than $80 p x$ tall has also been carried out. This height was chosen based on the fact that some pedestrian detection classifiers, the one based on deformable part models in particular, averagely perform better on close distance range pedestrians. 
TABLE IV

CLASSIFIER COMPARISON IN TERMS OF PRECISION (P) AND RECALL (R) ON SWIR IMAGES OVER ALL THE IMAGES

\begin{tabular}{|c|c|c|c|c|c|c|}
\cline { 2 - 7 } \multicolumn{1}{c|}{} & \multicolumn{2}{c|}{ Part-Models } & \multicolumn{2}{c|}{ Grammar-Models } & \multicolumn{2}{c|}{ HAAR } \\
\hline & $\mathrm{P}(\%)$ & $\mathrm{R}(\%)$ & $\mathrm{P}(\%)$ & $\mathrm{R}(\%)$ & $\mathrm{P}(\%)$ & $\mathrm{R}(\%)$ \\
\hline C & 64.89 & 57.10 & 67.38 & 38.77 & 63.51 & 7.1 \\
\hline F1 & 68.51 & 80.62 & 71.96 & 45.64 & 83.33 & 3 \\
\hline F2 & 41.21 & 87.13 & 79.30 & 64.19 & 81.05 & 6.5 \\
\hline
\end{tabular}

Results from this test are presented in fig. 9. Differences in performance using the grammar model-based detector are negligible, with improvements to be noticed only with the $\mathrm{C}$ filter acquired images. More improvements can be noticed using the part-based detector on the $\mathrm{C}$ filter acquired images although a drawback is shown on the F1 filter acquired sequence. So no significative changes in terms of performance were brought by uniforming the pedestrians' heights.

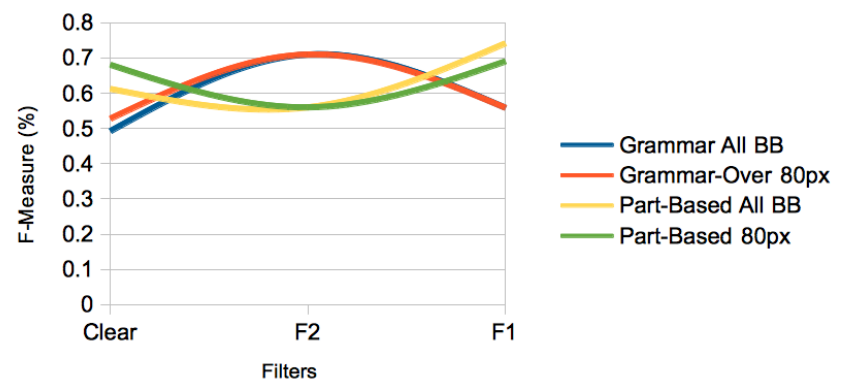

Fig. 9. Results comparison when testing on all the BB vs. BB surrounding pedestrians over $80 p x$ only.

\section{CONCLUSIONS}

Pedestrian detection is a fundamental requirement for ADAS systems and many efforts are dedicated to deal with this complex problem. Scientific investigations about the subject include exploring different approaches applied to images acquired in a single spectrum, most commonly the visible one, or adapting state-of-the-art approaches to other than the visible bandwidths.

In this paper we went through the second route, trying to understand if longer wavelengths as the SWIR ones providing clearer details at distance and different features from those appearing in visible images may be useful to deal with the detection of pedestrians. Despite some positive results, our tests on SWIR images in clear visibility conditions have shown that detection rates obtained are no better than those reported in state-of-the-art works involving visible only images datasets [26].

Further evaluations of the SWIR wavelengths should include night vision. Because the $\mathrm{O}-\mathrm{H}$ molecules floating in the upper atmosphere radiates energy at various intensities throughout the night, night vision on moonless nights is possible in the long wavelengths. These emissions enable night-time vision under the passive illumination of the sky making SWIR imagers very suitable for automotive applications as a valid alternative to current systems as cameras sensible to Near InfraRed wavelengths (NIR) or thermal cameras sensible to the Far InfraRed ones (FIR). Both these sort of devices in fact feature some important disadvantages: NIR cameras need special IR illuminators integrated in the vehicle to illuminate the area in front of it, whereas FIR cameras do not have this limitation but are still inherently expensive sensors for high resolution specifications. The SWIR technology can be considered somewhat in between these two extremes, featuring good resolution images at affordable prices for current automotive applications and at the same time showing wider ranges scenarios than NIR cameras benefiting of the night sky's natural infrared glare, which shines within the SWIR range.

\section{ACKNOWLEDGMENTS}

The work described in this paper has been developed in the framework of the 2WIDE_SENSE (WIDE spectral band \& WIDE dynamics multifunctional imaging SENSor Enabling safer car transportation) Project funded by EU within the FP7 Seventh Framework Programme.

\section{REFERENCES}

[1] P. Dollar, C. Wojek, B. Schiele, and P. Perona, "Pedestrian detection: An evaluation of the state of the art," Pattern Analysis and Machine Intelligence, IEEE Transactions on, vol. 34, no. 4, pp. 743-761, 2012.

[2] C. Keller, M. Enzweiler, and D. Gavrila, "A new benchmark for stereobased pedestrian detection," in Intelligent Vehicles Symposium (IV), 2011 IEEE. IEEE, 2011, pp. 691-696.

[3] T. Ogawa, H. Sakai, Y. Suzuki, K. Takagi, and K. Morikawa, "Pedestrian detection and tracking using in-vehicle lidar for automotive application," in Intelligent Vehicles Symposium (IV), 2011 IEEE. IEEE, 2011, pp. 734-739.

[4] C. Premebida, O. Ludwig, and U. Nunes, "Lidar and vision-based pedestrian detection system," Journal of Field Robotics, vol. 26, no. 9, pp. 696-711, 2009.

[5] B. Leibe, E. Seemann, and B. Schiele, "Pedestrian detection in crowded scenes," in Computer Vision and Pattern Recognition, 2005. CVPR 2005. IEEE Computer Society Conference on, vol. 1. IEEE, 2005, pp. $878-885$.

[6] D. M. Gavrila, "Pedestrian detection from a moving vehicle," in Computer Vision - ECCV 2000. Springer, 2000, pp. 37-49.

[7] M. Bertozzi, A. Broggi, A. Fascioli, and M. Sechi, "Shape-based Pedestrian Detection," in Procs. IEEE Intelligent Vehicles Symposium 2000, Detroit, USA, Oct. 2000, pp. 215-220.

[8] X. Liu and K. Fujimura, "Pedestrian detection using stereo night vision," Vehicular Technology, IEEE Transactions on, vol. 53, no. 6, pp. 1657-1665, 2004.

[9] M. Bertozzi, A. Broggi, A. Fascioli, T. Graf, and M.-M. Meinecke, "Pedestrian Detection for Driver Assistance Using Multiresolution Infrared Vision," IEEE Trans. on Vehicular Technology, vol. 53, no. 6, pp. 1666-1678, Nov. 2004, iSSN 0018-9545.

[10] M. Yasuno, S. Ryousuke, N. Yasuda, and M. Aoki, "Pedestrian detection and tracking in far infrared images," in Intelligent Transportation Systems, 2005. Proceedings. 2005 IEEE. IEEE, 2005, pp. 182-187.

[11] F. Suard, A. Rakotomamonjy, A. Bensrhair, and A. Broggi, "Pedestrian Detection using Infrared images and Histograms of Oriented Gradients," in Procs. IEEE Intelligent Vehicles Symposium 2006, Tokyo, Japan, June 2006, pp. 206-212.

[12] J. Li, W. Gong, W. Li, and X. Liu, "Robust pedestrian detection in thermal infrared imagery using the wavelet transform," Infrared Physics \& Technology, vol. 53, no. 4, pp. 267-273, 2010.

[13] A. Miron, B. Besbes, A. Rogozan, S. Ainouz, and A. Bensrhair, "Intensity self similarity features for pedestrian detection in far-infrared images," in Intelligent Vehicles Symposium (IV), 2012 IEEE. IEEE, 2012, pp. 1120-1125. 


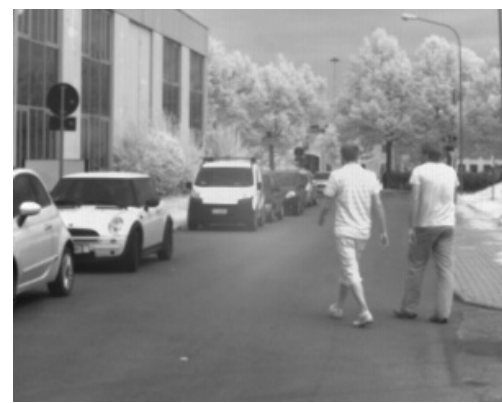

(a)

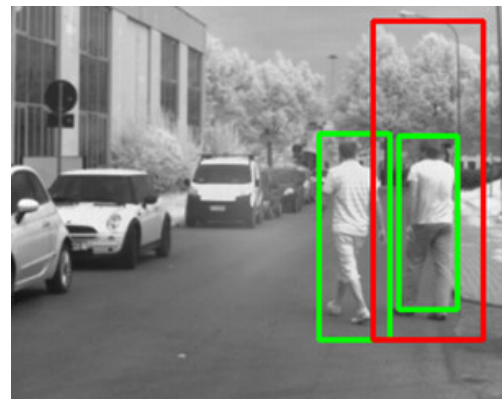

(d)

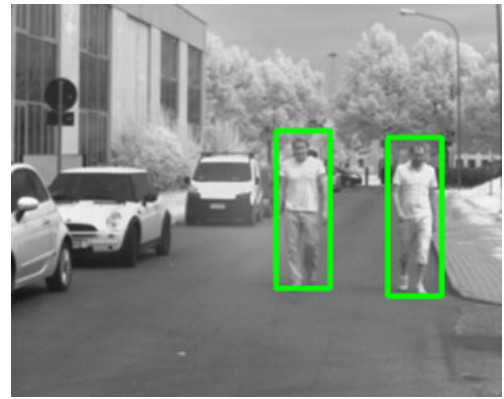

(g)

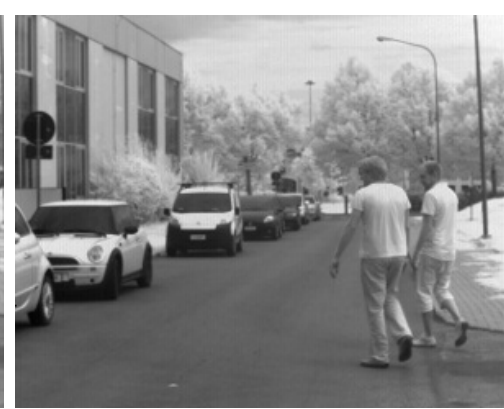

(b)

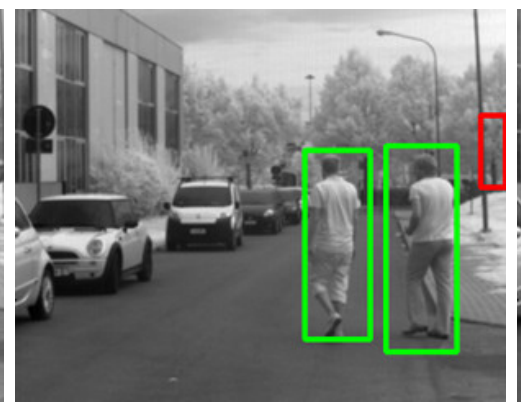

(e)

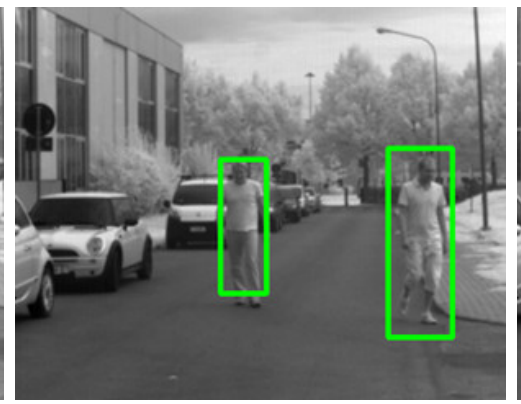

(h)

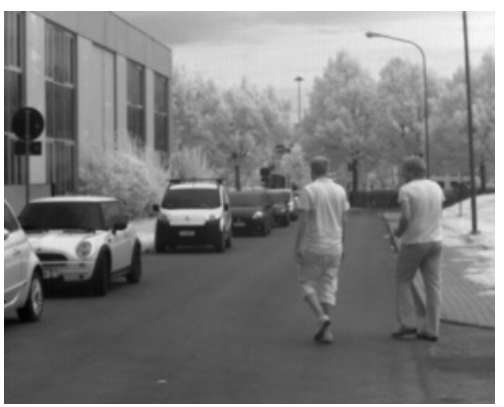

(c)

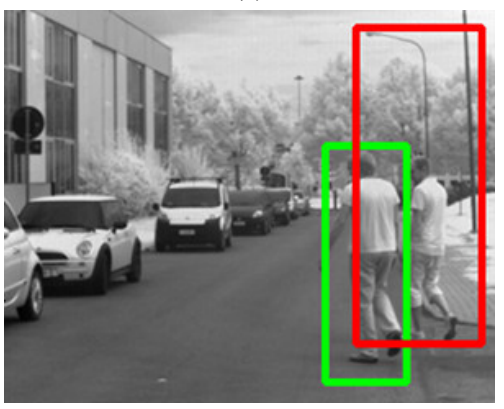

(f)

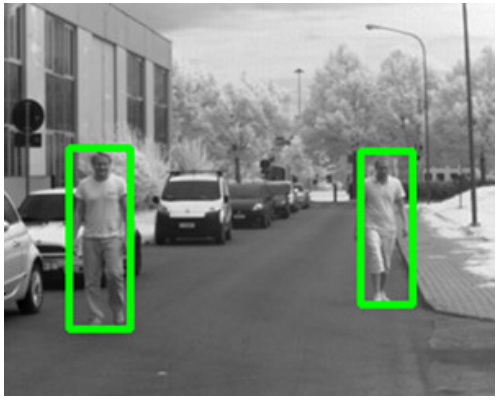

(i)

Fig. 10. Image examples from the sequences showing similar scenes and corresponding output results given by the grammar models: $\mathrm{C}$ filter range [a, d, g], F2 filter range [b, e, h] and F1 filter range [c, f, i]. False positives produced by the algorithm are surrounded by red BB while true positives are in green BB.

[14] D. Geronimo, A. M. Lopez, A. D. Sappa, and T. Graf, "Survey of pedestrian detection for advanced driver assistance systems," Pattern Analysis and Machine Intelligence, IEEE Transactions on, vol. 32, no. 7, pp. 1239-1258, 2010.

[15] M. P. Hansen and D. S. Malchow, "Overview of SWIR detectors, cameras, and applications," in Procs. SPIE 6939, Thermosense XXX, Mar. 2008.

[16] J. Valldorf and W. Gessner, Eds., Advanced Microsystems for Automotive Applications 2005. Berlin: Springer Verlag, June 2006, iSBN: 3540334092 .

[17] 2WideSense Website, "2WideSense EU Project," available at http://www.2wide-sense.eu.

[18] M. Bertozzi, R. I. Fedriga, A. Miron, and J.-L. Reverchon, "Pedestrian Detection in Poor Visibility Conditions: Would SWIR Help?" in Procs. IAPR Intl. Conf. on Image Analysis and Processing, Naples, Italy, Sept. 2013.

[19] P. F. Felzenszwalb, R. B. Girshick, and D. McAllester, "Cascade object detection with deformable part models," in Procs. IEEE Conf. on Computer vision and pattern recognition, 2010, pp. 2241-2248.

[20] P. F. Felzenszwalb, R. B. Girshick, D. McAllester, and D. Ramanan, "Object detection with discriminatively trained part-based models," IEEE Trans. on Pattern Analysis and Machine Intelligence, vol. 32, no. 9, pp. 1627-1645, 2010.

[21] R. Girshick, P. Felzenszwalb, and D. McAllester, "Object detection with grammar models," IEEE TPAMI, vol. 33, p. 12, 2011.
[22] R. Lienhart and J. Maydt, "An extended set of haar-like features for rapid object detection," in Image Processing. 2002. Proceedings. 2002 International Conference on, vol. 1. IEEE, 2002, pp. I-900.

[23] N. Dalal and B. Triggs, "Histograms of oriented gradients for human detection," in Computer Vision and Pattern Recognition, 2005. CVPR 2005. IEEE Computer Society Conference on, vol. 1. IEEE, 2005, pp. 886-893.

[24] P. Dollár, S. Belongie, and P. Perona, "The fastest pedestrian detector in the west," in British Machine Vision Conference, vol. 55, 2010.

[25] S. Walk, N. Majer, K. Schindler, and B. Schiele, "New features and insights for pedestrian detection," in Computer Vision and Pattern Recognition (CVPR), 2010 IEEE Conference on. IEEE, 2010, pp. 1030-1037.

[26] Caltech Pedestrian Detection Benchmark Website, "Caltech Pedestrian Detection Benchmark," available at http://www.vision.caltech.edu/Image_Datasets / CaltechPedestrians/. 